\title{
Bidirectionally-pumped remote L-band EDFA module utilizing stimulated Raman scattering
}

\begin{abstract}
This research was centered on an enhanced bidirectionally-pumped remote L-band erbiumdoped fiber amplifier (EDFA) module. The amplifier was backward-pumped using $1455 \mathrm{~nm}$ Raman laser while the SRS generated in the delivery line pumped the remote EDFA in the forward direction. Gain values around $12 \mathrm{~dB}$ and $9 \mathrm{~dB}$ were measured for input signal of 30 and $0 \mathrm{dBm}$ respectively. Excellent gain flatness was observed for both input levels with similar maximum gain variation of only $1.2 \mathrm{~dB} .30 \mathrm{dBm}$ input produced high noise figures up to 11.6 at the shorter wavelengths which declined as the signal moved further across the Lband window. The same trend was observed for $0 \mathrm{dBm}$ input where at $1570 \mathrm{~nm}, 12.1 \mathrm{~dB}$ noise figure was acquired and went down to $7.2 \mathrm{~dB}$ at $1605 \mathrm{~nm}$. Gain and noise figure behaviors were attributed to the gain competition due to the presence of the SRS, which was inadvertently amplified as well. The use of longer EDF length or careful selection of the pump wavelength can be considered to optimize the amplifier design.
\end{abstract}

Keyword: L-band; Amplifier; Pump wavelength; SRS; Gain competition; Gain flatness 